\title{
Direct Synthesis of Selenium Nanowire Mesh on a Solid Substrate and Insights into Ultrafast Photocarrier Dynamics
}

\author{
Wei Yan, ${ }^{\dagger, \#}$ Andrés Burgos-Caminal, ${ }^{\ddagger}, \#$ Tapajyoti Das Gupta, ${ }^{\dagger}$ Jacques-Edouard Moser, $^{\ddagger}$ \\ and Fabien Sorin*,†৫ \\ ${ }^{\dagger}$ Laboratory of Photonic Materials and Fibre Devices (FIMAP), Institute of Materials, École Polytechnique Fédérale de Lausanne,
CH-1015 Lausanne, Switzerland \\ ${ }^{\ddagger}$ Photochemical Dynamics Group, Institute of Chemical Sciences \& Engineering and Lausanne Centre for Ultrafast Science \\ (LACUS), École Polytechnique Fédérale De Lausanne, CH-1015 Lausanne, Switzerland
}

\section{Supporting Information}

\begin{abstract}
Selenium (Se) nanowires have generated much interest both for the fundamental understanding of crystal formation and growth and for technological applications in optoelectronics, imaging, piezoelectricity, catalysis, and energy harvesting and storage. Several methods have been established to synthesize Se nanowires, but they require sophisticated fabrication steps, are energy intensive, and may involve complex chemical reactions. Moreover, despite an increasing interest, little is known regarding photocarrier dynamics of Se nanowires. Here, we investigate a solution-based approach for the facile synthesis of single-crystal Se nanowires over the large scale where

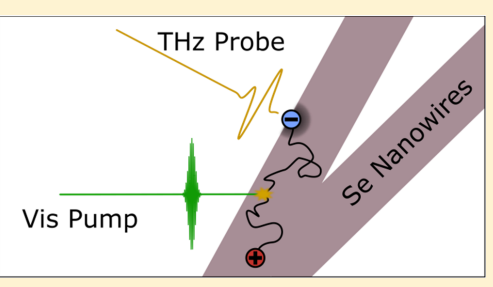
nanowires are directly grown from an amorphous bulk in a solution at room temperature without any chemical reaction. We study the nanowire nucleation and growth mechanism via electron microscopy. We also investigate, for the first time, the charge carrier dynamics and mobility of Se nanowire meshes by means of ultrafast transient absorption spectroscopy, nanosecond flash photolysis, and time-resolved terahertz spectroscopy. These contact-free and noninvasive approaches reveal a lifetime on the picosecond scale for free carriers and on the microsecond scale for trapped carriers, both of which are limited by trap-assisted recombination and a free carrier mobility of $\sim 3.0 \mathrm{~cm}^{2} \mathrm{~V}^{-1} \mathrm{~s}^{-1}$. Our work for the first time reveals the rationale behind the excellent properties of some Se NW-based optoelectronic devices. It also highlights the simplicity and robustness of the novel Se nanowire synthesis scheme and paves the way toward the simple fabrication of advanced nanowire-based electronic and optoelectronic nanodevices.
\end{abstract}

\section{INTRODUCTION}

One-dimensional semiconducting nanowires represent attractive building blocks for electronic and photonic devices ${ }^{1,2}$ such as transistors, ${ }^{3}$ optical devices, ${ }^{4}$ sensors, ${ }^{5,6}$ and metal-oxidesemiconductor circuits. ${ }^{7}$ The two typical fabrication paradigms of nanowires, i.e., top-down and bottom-up approaches, have become increasingly successful in making high-quality semiconducting nanowires. The simpler bottom-up approach, in particular, that relies on growth mechanisms such as vaporliquid-solid (VLS), solid-liquid-solid (SLS), or hydrothermal methods, ${ }^{5}$ provides a promising tool for the synthesis of nanowires in terms of cost, throughput, and complexity. VLS and SLS processes have been widely exploited to synthesize a rich variety of nanowires such as unitary, III-V and II-V semiconductors, oxides, carbides, nitrides, and phosphides. ${ }^{2}$ A number of solution-based methods have also been demonstrated to fabricate nanowires of silicon, III-V semiconductors, but also of less conventional materials for optoelectronics such as chalcogenides. ${ }^{8}$

Among the various chalcogenide materials, pure Se nanowires form an intriguing system for both fundamental studies and applied research. Interesting studies have been done based on Se nanowires (Se-NWs) in thermodynamics ${ }^{9}$ and crystal nucleation and growth. ${ }^{10,11}$ From an application point of view,
Se was one of the first investigated materials for its interesting electronic and optoelectronic properties. It indeed has a very strong absorption in the visible range and exhibits a good photoconductivity in its trigonal crystalline phase. Pure Se, ${ }^{12}$ as well as a variety of Se-based compounds, including chalcopyrite or kesterite, are commonly used in photodetecting $^{13-15}$ and photovoltaic devices. ${ }^{16,17}$ Crystalline Se also has excellent piezoelectric properties, ${ }^{11}$ and amorphous Se is being used as a scintillator for X-ray imaging. ${ }^{18}$ It is however difficult to grow Se in the right phase and with large and welloriented grains, ${ }^{19}$ which has rendered Se-based devices difficult to fabricate. The formation of nanowires however is a promising approach to alleviate this difficulty and control and optimize the microstructure of Se materials. Se-NWs have been investigated for high-speed photodetection, ${ }^{12}$ as efficient catalysts, $^{20}$ for solar cells, ${ }^{21}$ as fiber-based electronics, ${ }^{2,23}$ for high-performance lithium-ion batteries, ${ }^{24,25}$ and as chemical sensors. ${ }^{26}$

To fully exploit the potential of Se-NW-based devices, however, significant progresses must be made on two fronts:

Received: September 13, 2018

Revised: October 5, 2018

Published: October 9, 2018 


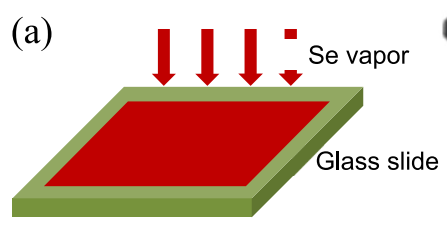

(i) Thin film deposition

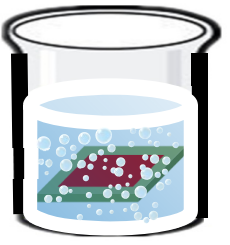

(ii) Sonication

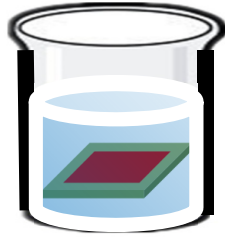

(iii) Soaking in 1-propanol

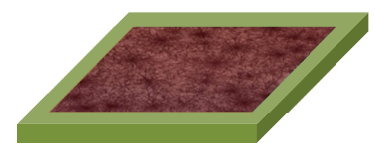

(iv) Nanowires formation
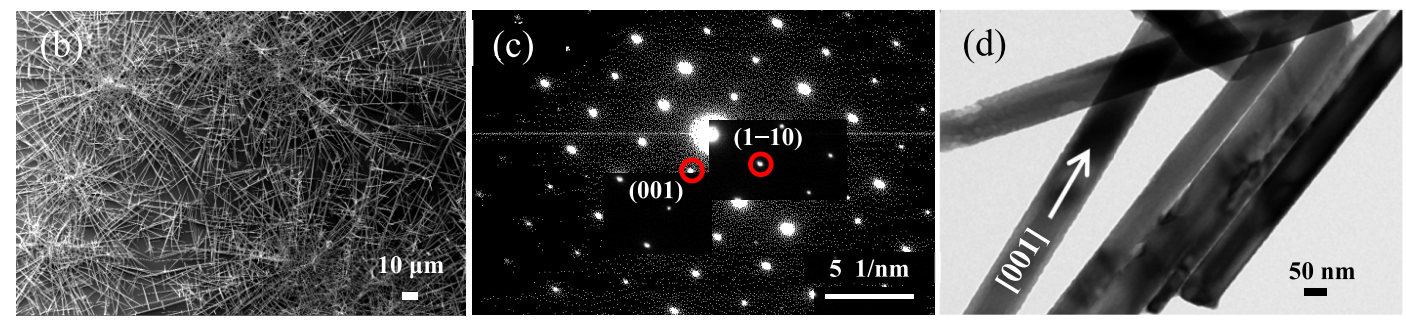

Figure 1. (a) Schematic of the synthesis process of Se nanowires. (b) SEM image of the nanowire network grown from a $75 \mathrm{~nm}$ thin film. (c) SAED pattern of the single nanowire in (d). (d) Bright-field TEM image of nanowires.

the deeper understanding of their photocarrier dynamics and their fabrication. First, despite the strong potential of Se-NWs, little is known concerning their charge carrier dynamics. Reviving the interest for, and improving the performance of, Se-based devices via the growth of NW entails a better characterization and understanding of Se charge carrier lifetime, mobility, and recombination mechanisms. Second, the fabrication of Se-NW remains poorly optimized to realize optical or optoelectronic devices, as they are either too complex or do not allow for the growth of nanowires from solid, patternable films directly on solid substrates. Vapor deposition techniques require the generation of Se vapors at high temperatures and the assistance of other materials. ${ }^{9,27}$ Hydrothermal techniques rely on decomposition of complex Se-based compounds at high temperatures in aqueous solutions via chemical reactions. ${ }^{10,11,26,28,29}$ All these techniques require complicated postsynthesis transferring methods for making Se nanowire-based devices. Byproducts formed during chemical reactions might also contaminate the nanowires, thereby affecting their practical applications. Simpler and efficient sonochemical methods have been proposed to grow nanowire from Se colloidal solutions. ${ }^{10}$ While simple and very efficient, this approach requires the use of liquid solution not well amenable to patterning nor to the direct growth on solid substrates of flat Se-NW meshes.

Here, we report on a newly developed solution-based approach for the synthesis of monocrystalline Se-NWs by leveraging a recent method to integrate semiconducting nanowire-based optoelectronic devices within functional polymeric fibers. ${ }^{12}$ Se-NWs are phase-transformed directly from a deposited amorphous Se thin film of controlled thickness, exposed to a solvent (here, 1-propanol) at room temperature without any chemical reaction. We demonstrate that Se-NWs can be synthesized on any solid substrate and over very large scale, even without an ultrasonic step. We further exploit this approach to develop a deeper understanding of both the growth mechanism and optoelectronic properties of Se-NWs from a sonochemical treatment. In particular, we first investigate nanowire nucleation and growth mechanisms by the systematic study of the effect of sonication and of the initial film thickness on the nanowire microstructure via optical and electron microscopy. Second, and for the first time, we investigate their charge carrier dynamics and mobility via advanced optical characterization techniques, namely, ultrafast transient absorption spectroscopy, nanosecond flash photolysis, and time-resolved terahertz spectroscopy. Our work sheds new light on the optical and electrical properties of Se nanowires, via the ability to probe charge carriers on an ultrafast time scale. It further reveals Se-NW as a promising alternative material platform for optoelectronic applications.

\section{EXPERIMENTAL SECTION}

Optical Microscopy and SEM and TEM Characterization. Optical micrographs were taken by a LEICA DM 2700 optical microscope (OM) 476 equipped with a LEICA MC170 HD camera. All the SEM samples were coated with a $10 \mathrm{~nm}$ carbon film. The SEM images were taken with a Zeiss Merlin field emission SEM (Zeiss, Göttingen, Germany) equipped with a GEMINI II column operating at $2.0 \mathrm{kV}$ with a probe current of $150 \mathrm{pA}$. The TEM images and SAED patterns were taken using a Talos F200X operating at $200 \mathrm{kV}$.

Time-Resolved Terahertz Spectroscopy Characterization. Three beams from the fundamental of a pulsed laser beam ( $45 \mathrm{fs}$ pulse duration, $\lambda=800 \mathrm{~nm}, 1 \mathrm{kHz}$ ) from an amplified Ti:sapphire laser (Libra, Coherent) were used for the time-resolved terahertz spectroscopy (TRTS) experiments. A first beam was employed to pump a white light seeded Optical parametric amplifier (OPerA Solo, Coherent) in order to generate the pump pulses centered at $500 \mathrm{~nm}$ for the pumpprobe experiment. The more powerful one of the two remaining beams (390 $\mu \mathrm{J}$ per pulse) was used to generate the probe beam, a short and broadband $\mathrm{THz}$ pulse, through a two-color plasma method. The beam was focused with a fused silica lens $(f=75 \mathrm{~mm})$, and the second harmonic was generated with a $100 \mu \mathrm{m} \mathrm{BBO}$ crystal. At the focal point the electric field of the two-color beam was strong enough to form a plasma filament that radiates a broadband $\mathrm{THz}$ pulse $(200 \mathrm{fs}$, $1-20 \mathrm{THz}$ ) that was subsequently collimated and focused with parabolic mirrors onto the sample. The transmitted beam went through two additional parabolic mirrors toward a homemade $\mathrm{ABCD}$ detector (Air Biased Coherent Detection ${ }^{30}$ ). The remaining beam (40 $\mu \mathrm{J} /$ pulse) was used as a gate for the 
detection, generating a second harmonic signal proportional to the $\mathrm{THz}$ electric field measured with a PMT (PMM01, Thorlabs). The samples were specially grown on a flat HDPE substrate completely transparent to our broadband $\mathrm{THz}$ pulse.

Ultrafast Transient Absorption Spectroscopy Characterization. A different chirped pulse amplified Ti:sapphire laser (CPA-2001, Clark-MXR) was used to generate the pump and probe beams for the ultrafast transient absorption spectroscopy (TAS) characterization. The fundamental of the laser (120 fs pulse duration, $\lambda=778 \mathrm{~nm}, 1 \mathrm{kHz}$ ) was used to pump a two-stage noncolinear optical parametric amplifier (NOPA plus, Clark MXR) to generate the pump beam with a central wavelength of $520 \mathrm{~nm}$. The output of the NOPA was then compressed to $\sim 50$ fs with a pair of SF10 prisms, chopped at $500 \mathrm{~Hz}$, set at a magic angle $\left(54.7^{\circ}\right)$ with respect to the probe, and sent into the sample. The probe beam was obtained through white light generation in $\mathrm{a} \mathrm{CaF}_{2}$ crystal. In order to obtain the $\Delta A$ signal, the probe beam was divided into a signal beam and focused on the sample with an off-axis parabolic mirror and a reference beam. Both were spectrally resolved using spectrographs (Princeton instruments, Spectra Pro 2150i) and CCD detectors (Hamamatsu, S7030-0906).

Nanosecond Flash Photolysis Characterization. A frequency doubled Q-switched Nd:YAG laser (NT-342, Ekspla) was used as a pulsed pump (5 ns, $532 \mathrm{~nm}, 20 \mathrm{~Hz}$ ) for the nanosecond flash photolysis (NFP) experiments. The probe light was provided by a halogen lamp and detected with a PMT (R9910, Hamamatsu) after a monochromator. Finally, the time-resolved signal was recorded and digitized with an oscilloscope (DPO 7254, Tektronix).

\section{RESULTS AND DISCUSSION}

As schematically shown in Figure 1a, the approach we developed starts with thermally depositing a Se thin film with a desired thickness on a substrate, here a glass slide. The as-deposited Se, rapidly quenched on the substrate, is in an amorphous state after deposition. Subsequently, the sample was sonicated in distilled water for $1 \mathrm{~min}$ before it was soaked in 1-propanol for varying times (a few hours to a few days), adjustable depending on the thickness of the film. 1-Propanol is chosen because it dissolves the amorphous Se phase. Finally, the amorphous bulk Se transformed into a mesh of nanowires (Figure 1a). The whole process was accomplished at room temperature and ambient condition. The scanning electron microscopy (SEM) image of a sample $(75 \mathrm{~nm}$ thin film) soaked for $10 \mathrm{~h}$ shown in Figure $1 \mathrm{~b}$ reveals that a dense array of Se-NWs was synthesized on a $75 \times 25 \mathrm{~mm}^{2}$ glass slide. Note that with our approach Se-NWs can be synthesized on any substrate and over a large scale. The selected-area electron diffraction (SAED) from the transmission electron microscope (TEM) characterization (Figure 1c) of a typical nanowire (Figure 1d) indicates that it is monocrystalline. The pattern can be indexed as the trigonal phase, where the lattice constants calculated from the pattern $(a=0.433 \mathrm{~nm}, c=0.490$ $\mathrm{nm}$ ) match well with the ones of the Se trigonal phase reported in the literature $(a=0.436 \mathrm{~nm}, c=0.495 \mathrm{~nm}) .^{31}$ The nanowires preferentially grow along the [001] direction. Several diffraction patterns taken from different regions of an individual nanowire showed the same crystal orientation, indicating that nanowires exhibit monocrystalline nature without grain boundaries. This growth and its specific orientation are consistent with our previous theoretical analysis for Se-NWs synthesized in multimaterial fibers. ${ }^{12}$ Atomistic simulations indeed revealed that the presence of the solvent exacerbates the interfacial energy anisotropy between the basal plane $((001))$ and the prismatic planes of Se crystals. This anisotropy favors one-dimensional nanowire growth because a crystal plane grows at a rate proportional to its respective interfacial energy, from the thermodynamic point of view. ${ }^{32}$

In order to further understand the nanowire formation mechanism, we first studied the effect of sonication. Figure $2 \mathrm{a}, \mathrm{b}$ shows the microstructure of the sample $(75 \mathrm{~nm}$ thin film)
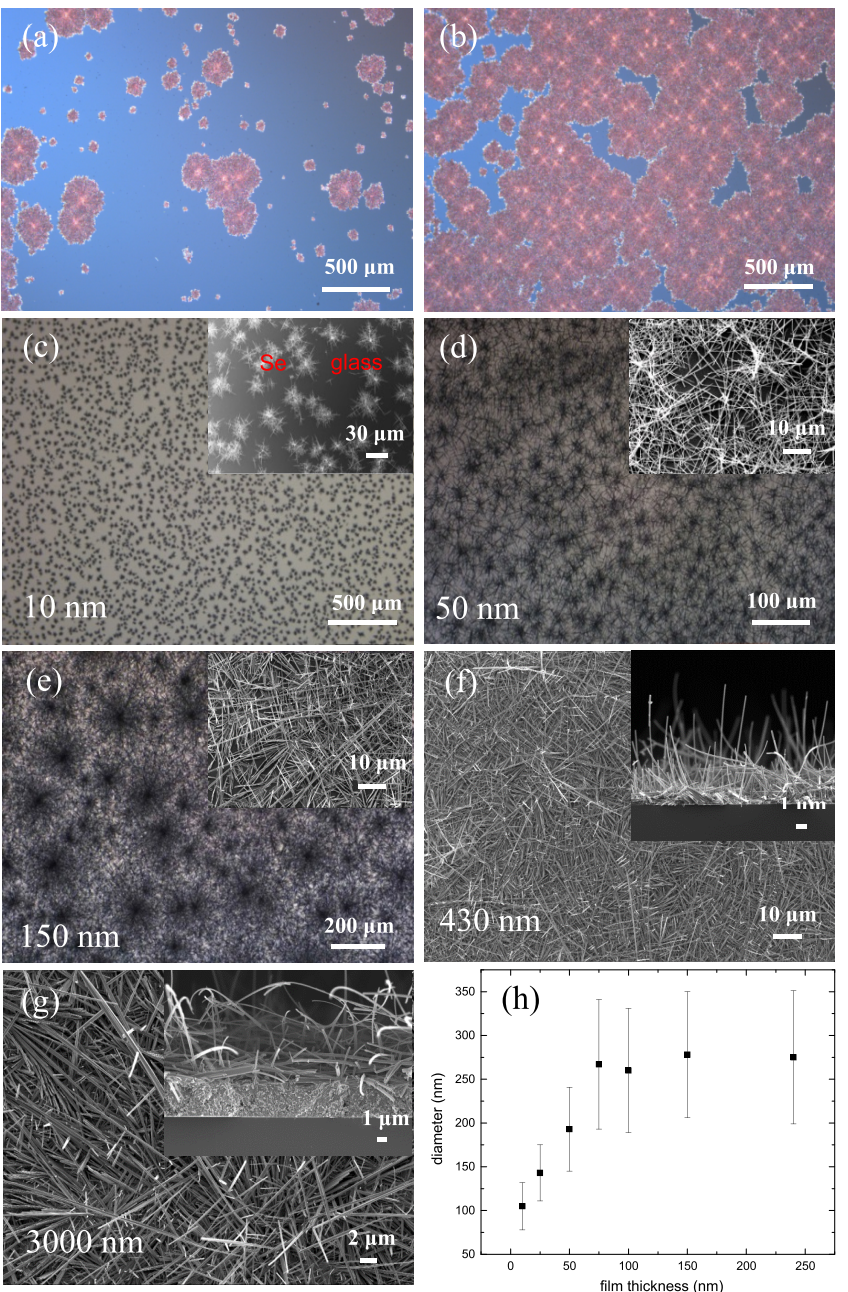

Figure 2. (a, b) Optical photograph of the nanowires grown from a 75 $\mathrm{nm}$ thin film without and with sonication treatment, respectively. Both of the samples were soaked for $10 \mathrm{~h}$. (c-e) Optical photograph of the nanowires grown from 10, 50, and $150 \mathrm{~nm}$ thin films, respectively. (f,g) SEM images of the nanowires grown from 430 and $3000 \mathrm{~nm}$, respectively. The insets in $\mathrm{c}-\mathrm{g}$ are corresponding crosssectional SEM images. (h) The diameter of nanowires as a function of the thin-film thickness.

without and with sonication after $10 \mathrm{~h}$ of solvent exposure, respectively. When the sample was sonicated the number of nanowire clusters was significantly higher compared to nonsonicated samples. It appears that sonication is not necessarily required when the nanowires are grown directly from a solid thin film, but it can accelerate nucleation. During sonication indeed, a large number of bubbles form, and their collapse induced by cavitation produces intense local heating (exceeding $5000 \mathrm{~K}$ ) and high pressure (exceeding $500 \mathrm{~atm}$ ), ${ }^{33}$ which causes rapid heterogeneous nucleation. Without 

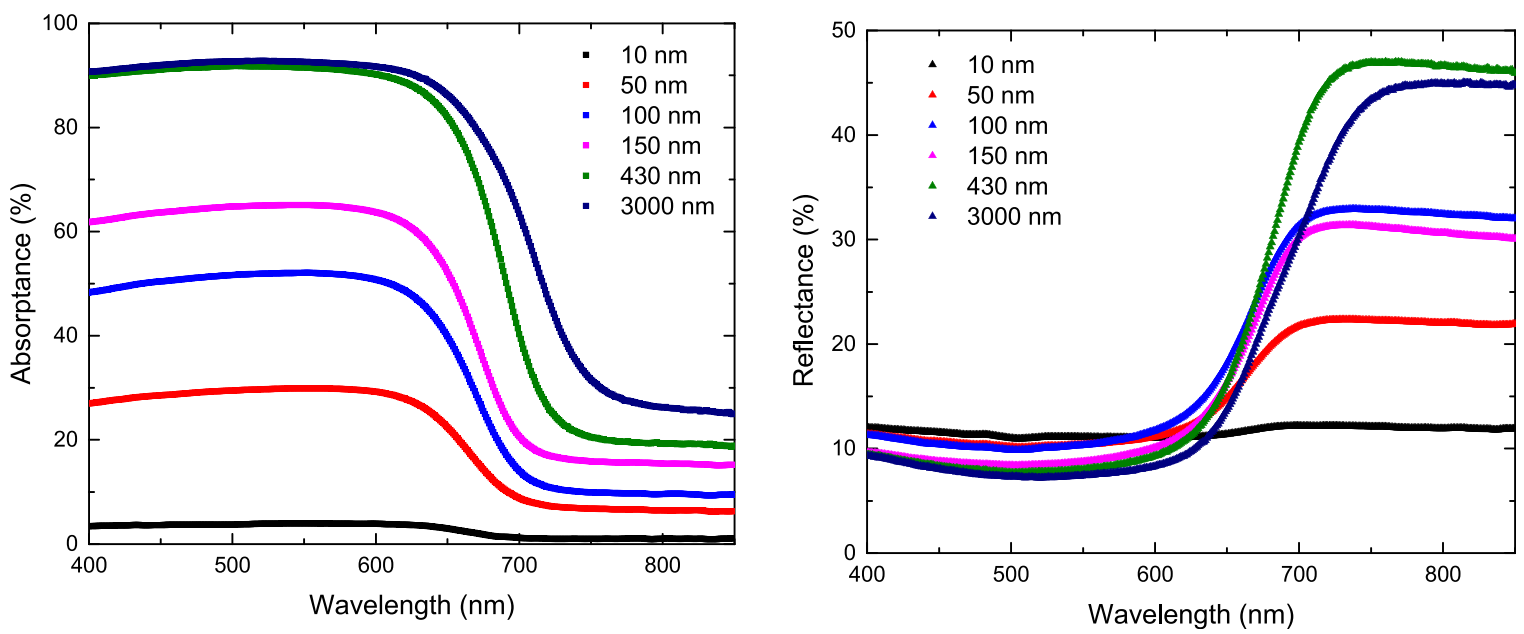

Figure 3. Visible and near-infrared absorptance and reflectance spectra of nanowire samples grown from 10, 50, 100, 150, 430, and 3000 nm thin films.

sonication, nucleation also happened in the sample following the formation of a supersaturated solution due to the solubility of amorphous Se in 1-propanol. This created a nonequilibrium condition for the system which resulted in the aggregation of dissolved atoms and eventually led to the formation of nuclei that acted as centers of crystallization. Yet, the number of nuclei formed in solution is lower compared to a sonication treatment.

We now turn to the influence of the thickness of the initial thin film on the microstructure of the nanowires and the mesh properties. Se thin films of varying thickness from 10 to 3000 $\mathrm{nm}$ were deposited on glass slides. Figure $2 \mathrm{c}-\mathrm{f}$ shows the microstructure of the nanowire mesh grown from the different thin films. It appears that all the amorphous films transformed into nanowires in a sufficient soaking time. After the nuclei have formed, the Se atoms dissolved in the solvent are more energetically favored to attach to these crystalline nuclei, resulting in their growth after they reached a critical size. The randomly separated nanowire clusters in the inset of Figure $2 c$ result from the heterogeneous distribution of the nuclei after sonication. Because the growth rate of the basal plane is higher than that of prismatic planes, crystals grow into nanowires with sharp prismatic tips, as indicated by the cone shape of the nanowires in Figure 2. Macroscopically, crystal growth was anisotropic, leading to the snowflake-like clusters consisting of dendritic branches. The maximum nanowire diameter for the $10 \mathrm{~nm}$ sample is below $150 \mathrm{~nm}$ (Figure 2c). When increasing the thickness of the film, the snowflake-like clusters become bigger and denser. The maximum diameter of each nanowire however increases first before reaching a plateau for the samples above a $75 \mathrm{~nm}$ thickness as demonstrated by the SEM images of the samples (430 and $3000 \mathrm{~nm}$ ) in Figure 2f,g and a statistic analysis of the maxium diameter of each wire shown in Figure $2 \mathrm{~h}$. The statistics of the ratio of the nanowire length to diameter (shown in the inset of Figure 2f) were measured to be centered at 25 , which agrees very well with our simulated lattice constant ratio (ch/ah) from first-principles densityfunctional theory calculations. ${ }^{12}$ For thick samples, not all the film was transformed into nanowires. For the thickest sample $(3000 \mathrm{~nm})$, soaked in 1-propanol for 5 days, the cross-sectional SEM image (the inset in Figure $2 \mathrm{~g}$ ) indicates a composite structure consisting of the nanowire array growing on a film that remained amorphous. The nanowire mesh at the top of the remaining film maintained the same thickness even when the sample was treated for a longer soaking time (e.g., 10 days), which implies that the solvent may not penetrate into the film below the mesh and atom diffusion becomes more difficult when the nanowire mesh is thick enough. A critical thickness below which all the film can transform into nanowires was consistently found to be $\sim 430 \mathrm{~nm}$ (Figure $3 \mathrm{~g}$ ).

We then investigated the optical response of the nanowire samples by measuring the optical absorption and reflection spectra in the visible and near-infrared regions, as shown in Figure 3. We first note a strong absorption and low reflection in the visible region, due to an antireflection effect from the porous nanowire mesh and light trapping likely occurring. The onset of absorption agrees with an indirect band gap at around $1.85 \mathrm{eV}$ and a direct one at $1.95 \mathrm{eV}$ reported in the literature. ${ }^{34,35}$ In addition, leaky waveguide modes of individual nanowires may also contribute to the absorption. ${ }^{36}$ The distribution of midgap states, enhanced by the increased surface area, creates a well-known tail of absorption below the band gap. ${ }^{37}$ Overall, absorption increases with increasing thickness as expected, and it plateaus at around $90 \%$ above 430 $\mathrm{nm}$ of initial Se thickness owing to the $10 \%$ of reflectance. As explained above, a thicker film does not provide a denser nanowire mesh, and the optical absorption is dominated by the mesh in the visible below $670 \mathrm{~nm}$. Above this value, the underlying Se film that did not transform into nanowires contributes to a slight increase of absorption. The nanowire mesh however dictates the optical reflectance across the whole spectrum, with a low reflection below around $670 \mathrm{~nm}$ that increases with wavelength as the mesh is denser.

We now turn to the characterization of charge carrier dynamics of the nanowire network using a combination of three time-resolved spectroscopy techniques, i.e., time-resolved terahertz spectroscopy (TRTS), ultrafast transient absorption spectroscopy (TAS), and nanosecond flash photolysis (NFP). More details about the three techniques are given in the Experimental Section. We first probed the nanowires with ultrafast TRTS and TAS on the picosecond time scale. TRTS relies on the measurement of the change in transmission of the terahertz electric field through the sample, which can be directly related to the photoconductivity. ${ }^{38}$ In order to improve the signal, the thickness of the nanowire mesh for the TRTS characterization can be controlled to completely absorb the 

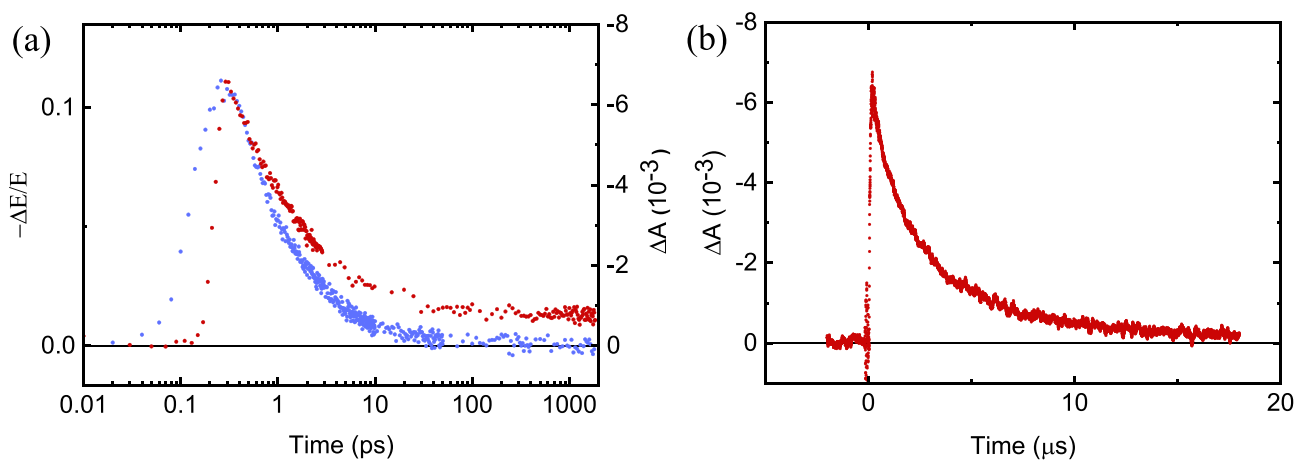

Figure 4. (a) Time dependence of the photoconductivity and the difference of absorption $(\Delta A)$ measured by the TRTS (blue, $\lambda_{\text {ex }}=500 \mathrm{~nm}, F=$ $426 \mu \mathrm{J} \mathrm{cm}^{-2}$ ) and TAS (red, $\lambda_{\mathrm{obs}}=650 \mathrm{~nm}, \lambda_{\mathrm{ex}}=520 \mathrm{~nm}, F=96 \mu \mathrm{J} \mathrm{cm}^{-2}$ ) techniques for the nanowires grown from $400 \mathrm{and} 200 \mathrm{~nm}$ thin films, respectively. (b) Time dependence of the difference of absorption $(\Delta A)$ measured by the long-lived NFP technique $\left(\lambda_{\mathrm{ex}}=532 \mathrm{~nm}, F=211 \mathrm{~mJ}\right.$ $\mathrm{cm}^{-2}$ ) for the nanowires grown from a $200 \mathrm{~nm}$ thin film.

pump pulses (here, $\lambda=500 \mathrm{~nm}$ ), thereby maximizing charge carrier generation without the need to increase the fluence of the pump pulse. Hence, a nanowire sample grown from a 400 $\mathrm{nm}$ thin film that exhibits the highest absorption (Figure 3) was used for this measurement. Alternatively, TAS records the difference of absorption spectra $(\Delta A)$ between the excited sample and the sample in the ground state. Thus, the sample must be partially transparent to the probe pulse. A nanowire sample grown from a $200 \mathrm{~nm}$ thin film was used for this measurement.

As shown in Figure 4a, a rapid rise in photoconductivity and $\Delta A$ in the respective signal, limited by the time resolution of the instrument, is observed after the photoexcitation due to the generation of carriers. A very fast multiexponential decay in the order of a few ps is readily observed after the rise with both methods. The faster part of the signal decay (90\% of the TRTS one) can be fitted with a biexponential equation (see the equation in the SI), obtaining lifetimes in the order of $\tau_{1}=0.5$ ps and $\tau_{2}=3$ ps. These carrier lifetimes compare favorably with that of some silicon, GaAs, and GaAs/AlGaAs core-shell nanowires. ${ }^{39}$ There is a clear differentiation between the signals that can be obtained with the two techniques. In TRTS, unless a major transition corresponding to a low energy vibrational mode is strongly affected, the photoconductivity is responsible for an increased absorption of THz. This photoconductivity arises from the introduction of new charge carriers and depends on their density and mobility. ${ }^{40}$ Thus, a decrease in $\mathrm{THz}$ absorption over time can be attributed to carrier recombination or a decrease in mobility or both. In TAS, any excited-state species that produces a change in the absorption profile in the visible region can be observed. In fact, the observed TAS signal in Figure $4 \mathrm{a}$ is a ground state bleaching (GSB, $\Delta A<0)$ near the band gap $(650 \mathrm{~nm})$. Any process that depletes the electron population capable of undergoing the transition with the corresponding energy will cause such a bleaching. This includes the formation of free carriers due to band filling ${ }^{41}$ but also immobilized trapped carriers $^{42,43}$ and excitons. ${ }^{44,45}$ Overall, the observed decay in both measurements is very similar. However, in TRTS we obtain a complete recovery of the initial signal, while in TAS a certain offset bleaching is maintained. If the fundamental difference between the signals obtained with the two experiments is taken into account, it can be concluded that most carriers rapidly return to the ground state, while a small portion stays in a state that gives rise to no or negligible $\mathrm{THz}$ absorption but allows a GSB. Trapped carriers are believed to fit in this description as they exhibit a greatly reduced mobility while maintaining a GSB, as has been reported before. ${ }^{46}$ In order to further explore this possibility, fluence-dependent TRTS measurements were carried out. The results (Table S1, Supporting Information) show little change in recombination lifetimes when changing the fluence, except at lower fluences. Note that trigonal selenium $(\mathrm{t}-\mathrm{Se})$ is an indirect semiconductor $^{47}$ that disfavors bimolecular recombination, and the sample is a nanowire mesh that exhibits a significantly enhanced density of midgap states (traps) due to the increased surface to volume ratio. It can therefore be concluded that the main recombination pathway is trap-assisted recombination (Shockley-Read-Hall) $^{48}$ instead of bimolecular recombination that is highly dependent on the excitation intensity.

In order to probe the nature of these trapped carriers, we then performed NFP measurements on a microsecond scale. Figure $4 \mathrm{~b}$ shows that these trapped carriers can last up to tens of microseconds before completely recombining. Biexponential fits yielded lifetimes of $\tau_{1}=0.9 \mu \mathrm{s}$ and $\tau_{2}=4.1 \mu \mathrm{s}$. Fluencedependent NFP measurements shown in the Supporting Information (Table S2) again indicate that carrier lifetime is not dependent on the incident flux, which further confirms the trap-assisted recombination mechanism. The microsecond time scale of these trapped carriers in our trigonal Se-NWs is comparable to that of mobile carriers recently reported in trigonal Se powders measured utilizing the transient microwave conductivity technique. ${ }^{49}$ The recovery time on the picosecond time scale measured by TRTS can then be attributed to ultrafast trapping of carriers mediated by surface states of each individual nanowire and contacts between nanowires, given the large surface to volume ratio of these randomly arranged samples. Similar dramatic changes in carrier dynamics due to increased surface states have been observed before, e.g., in nanoporous $^{42}$ and microcrystalline ${ }^{50}$ silicon. Therefore, a clear effect of the enhanced nanowire aspect ratio has been observed, heavily enhancing monomolecular recombination. Nevertheless, the trapped carriers in current Se nanowires exhibit such a long lifetime as measured by NFP that they can advance and be collected via hopping transport before they annihilate. This is beneficial for the use of Se-NWs in optoelectronic devices and is at the heart of the fiber-integrated device performance we have demonstrated in a recent work. ${ }^{12}$

Next, we studied the carrier mobility of the nanowire network. The TRTS measurements were additionally used to calculate the short-range mobility of charge carriers. Indeed, TRTS probes the intrinsic mobility on a short length scale, 
typically $<100 \mathrm{~nm}$, due to the short duration of the electric field cycle. ${ }^{38}$ The frequency-averaged photoconductivity $\Delta \sigma$ was obtained using the maximum electric field point of the single-cycle $\mathrm{THz}$ pulse following eq 1

$$
\Delta \sigma=-\frac{\left(n_{\mathrm{air}}+n_{\mathrm{HDPE}}\right) \varepsilon_{0} c}{L} \frac{\Delta E}{E}
$$

where $n_{\text {air }}$ and $n_{\text {HDPE }}$ are the refractive indices of air and the HDPE (completely transparent to our broadband $\mathrm{THz}$ pulse) substrate, respectively; $\varepsilon_{0}$ is the free space permittivity; $c$ is the speed of light; and $\Delta E$ is the photoinduced change in the transmitted $\mathrm{THz}$ electric field while $E$ is the initial one. Lastly, $L$ is the sample thickness. Here, we consider an effective thickness $\left(L_{\mathrm{e}}\right)$ corresponding to a compact film of $\mathrm{t}-\mathrm{Se}$ nanowires, in order to obtain an average mobility over the probed ensemble. The average mobility can then be calculated with $^{51}$

$$
\mu=\frac{\Delta \sigma}{N e}
$$

where $N$ is the charge carrier density and $e$ the elementary charge. In order to estimate $N$ we assume a complete photon to charge carrier conversion obtaining two carriers (electron and hole) per photon at time zero. Since the photoconductivity presents a very fast decay, being nonnegligible within the time duration of the pump pulse, the peak signal has to be extracted. A straightforward method is to use the amplitudes of the exponential decays convoluted with gaussians used for the fit (Supporting Information). These amplitudes correspond to the initial signal that would be observed after a delta function excitation pulse.

$N$ is calculated as $N=f_{\text {abs }} N_{\mathrm{p}} \phi$, where $f_{\text {abs }}$ is the absorptance measured with the integrating sphere, subtracting the amount of absorptance obtained at $850 \mathrm{~nm}$ to the one at $\lambda_{\text {ex }} ; \phi$ is the carrier generation yield that we assume to be equal to 2 ; and $N_{\mathrm{p}}$ is the average photon density on the sample volume (where $L_{\mathrm{e}}$ is present). Therefore, $L_{\mathrm{e}}$ cancels out in the calculation of $\mu$, yielding it irrelevant within the approximations used. Taking advantage of the mobility measurements that do not progressively vary at different fluences we reach a value of $\mu$ $=2.98 \pm 0.42 \mathrm{~cm}^{2} \mathrm{~V}^{-1} \mathrm{~s}^{-1}$ (Table 1$)$. The mobility value

Table 1. Fluence-Dependent TRTS Measurements of Carrier Mobility

\begin{tabular}{cccc}
$\begin{array}{c}\text { fluence } \\
\left(\mu \mathrm{J} \mathrm{cm}^{-2}\right)\end{array}$ & $\begin{array}{c}\text { total absorbed photon density } \\
\left(\mathrm{m}^{-2}\right)\end{array}$ & $-\Delta E / E$ & $\begin{array}{c}\mathrm{mob} \\
\left(\mathrm{cm}^{2} \mathrm{~V}^{-1} \mathrm{~s}^{-1}\right)\end{array}$ \\
71 & $1.77 \times 10^{18}$ & 0.0305 & 3.60 \\
142 & $3.31 \times 10^{18}$ & 0.041 & 2.59 \\
227 & $5.30 \times 10^{18}$ & 0.0803 & 3.17 \\
284 & $6.63 \times 10^{18}$ & 0.104 & 3.28 \\
426 & $9.94 \times 10^{18}$ & 0.136 & 2.88 \\
568 & $1.33 \times 10^{19}$ & 0.150 & 2.37 \\
\hline
\end{tabular}

obtained here for the Se nanowire network is much higher than that of a single Se nanowire synthesized by the hydrothermal technique measured using a contact probe technique. ${ }^{52}$ The latter technique probes the mobility of carriers as they travel between two electrodes over long time scales. Thus, they have time to get trapped and scatter on different defects found along the way. On the contrary, in our TRTS measurements the carriers are probed over small distances before they equilibrate with defect trapping states. The mobility obtained here can therefore represent an upper limit for the Se nanowire network. Interestingly, the mobility value is higher than that of polycrystalline Se powders, ${ }^{39}$ while it is lower than that of bulk Se single crystals. ${ }^{53}$ It should be noted that the current carrier mobility measured by TRTS is an average value for the Se nanowire network where each individual wire has a random orientation with respect to the electric field of THz. However, even though experimental uncertainties and approximations can contribute to the differences, we believe that grain boundaries in polycrystalline samples, surface states and interconnections in nanowire samples, and crystal orientation can strongly affect carrier mobility. ${ }^{54}$ Indeed, the mobility must be higher along the helical chains of trigonal selenium than crossing them. Thus, bulk single crystals without grain boundaries, surface states, or interconnections, probed along the (001) orientation, exhibit the highest mobility. Finding ways to reduce the density of interconnections such as aligning the nanowire mesh via electric forces or thermal gradients would allow an increase in carrier mobility and therefore enhance device performance. This has been demonstrated by our recent work where engineering the number of defects in the Se nanowire network significantly improves the electronic and optoelectronic performance in terms of photoresponsivity, photosensitivity, and response speed. ${ }^{12}$ Surface passivation could also be exploited to unpin the Fermi level from the conduction band and therefore undermine surface scattering and yield enhanced carrier mobilities approaching bulk values, while reducing surface recombination.

\section{CONCLUSIONS}

In summary, we have developed a novel and simple solutionbased approach for the large-scale synthesis of Se single-crystal nanowires directly from amorphous Se thin films. Nanowire nucleation and growth mechanisms were elucidated via electron microscopy combining our previous atomistic simulation, which provides guidance for the nanowire transformation of other anisotropic systems, such as $\mathrm{Te}$ or $\mathrm{Se} / \mathrm{Te}$ compounds. With our approach, Se-NWs can be potentially synthesized on any substrates that are flexible, soft, stretchable, foldable, or wearable. It also facilitates the fabrication of Se-NW-based electronics and optoelectronics with the optimal microstructure and without the need for transferring methods. For the first time, we investigated the charge carrier dynamics and mobility of Se-NWs using a timeresolved contact-free and noninvasive approach, revealing picosecond-long free carrier lifetime and microsecond-long trapped carrier lifetime and a free carrier mobility of $\sim 3.0 \mathrm{~cm}^{2}$ $\mathrm{V}^{-1} \mathrm{~s}^{-1}$. Carriers recombine via a trap-assisted recombination mechanism. In spite of a high density of surface states that influence carrier mobility, Se in the form of nanowires exhibits an excellent combination of efficient light absorption, long carrier lifetime, good mobility, easy synthesis, and processability, making them of interest for high-performance electronic and optoelectronic devices.

\section{ASSOCIATED CONTENT}

\section{S Supporting Information}

The Supporting Information is available free of charge on the ACS Publications website at DOI: 10.1021/acs.jpcc.8b08942.

We discuss the models we used to fit the time dependence of the photoconductivity and the difference of absorption measured by the time-resolved terahertz 
spectroscopy (TRTS) and ultrafast transient absorption spectroscopy (TAS), as well as time dependence of the difference of absorption measured by nanosecond flash photolysis (NFP), fluence-dependent TRTS measurements of carrier lifetime, and fluence-dependent NFP measurements of carrier lifetime (PDF)

\section{AUTHOR INFORMATION}

\section{Corresponding Author}

*E-mail: fabien.sorin@epfl.ch.

\section{ORCID}

Fabien Sorin: 0000-0003-1019-6484

\section{Author Contributions}

${ }^{\#}$ W. Yan and A. Burgos-Caminal contributed equally to the work.

\section{Notes}

The authors declare no competing financial interest.

\section{ACKNOWLEDGMENTS}

W.Y. and F.S. would like to acknowledge the Swiss National Science Foundation (Grant No. 200021 146871) and the European Research Council (ERC Starting Grant 679211 "FLOWTONICS") for their funding support. They also acknowledge the support of the Swiss CCMX Materials Challenge funding scheme. A.B.C. and J.E.M. are grateful to the SNSF (Grant No. 200021_175729) and to the National Center of Competence in Research "Molecular Ultrafast Science and Technology" (NCCR-MUST), a research instrument of the SNSF, for financial support.

\section{REFERENCES}

(1) Gudiksen, M. S.; Lauhon, L. J.; Wang, J.; Smith, D. C.; Lieber, C. M. Growth of Nanowire Superlattice Structures for Nanoscale Photonics and Electronics. Nature 2002, 415, 617-620.

(2) Li, Y.; Qian, F.; Xiang, J.; Lieber, C. M. Nanowire Electronic and Optoelectronic Devices. Mater. Today 2006, 9, 18-27.

(3) Xiang, J.; Lu, W.; Hu, Y.; Wu, Y.; Yan, H.; Lieber, C. M. Ge/Si Nanowire Heterostructures as High-Performance Field-Effect Transistors. Nature 2006, 441, 489-493.

(4) Johnson, J. C.; Choi, H.-J.; Knutsen, K. P.; Schaller, R. D.; Yang, P.; Saykally, R. J. Single Gallium Nitride Nanowire Lasers. Nat. Mater. 2002, 1, 106-110.

(5) Pauzauskie, P. J.; Yang, P. Nanowire Photonics. Mater. Today 2006, 9, 36-45.

(6) Cui, Y.; Wei, Q.; Park, H.; Lieber, C. M. Nanowire Nanosensors for Highly Sensitive and Selective Detection of Biological and Chemical Species. Science 2001, 293, 1289-1292.

(7) Nam, S.; Jiang, X.; Xiong, Q.; Ham, D.; Lieber, C. M. Vertically Integrated, Three-Dimensional Nanowire Complementary MetalOxide-Semiconductor Circuits. Proc. Natl. Acad. Sci. U. S. A. 2009, 106, 21035-21038.

(8) Law, M.; Goldberger, J.; Yang, P. Semiconductor Nanowires and Nanotubes. Annu. Rev. Mater. Res. 2004, 34, 83-122.

(9) Ren, L.; Zhang, H.; Tan, P.; Chen, Y.; Zhang, Z.; Chang, Y.; Xu, J.; Yang, F.; Yu, D. Hexagonal Selenium Nanowires Synthesized via Vapor-Phase Growth. J. Phys. Chem. B 2004, 108, 4627-4630.

(10) Mayers, B. T.; Liu, K.; Sunderland, D.; Xia, Y. Sonochemical Synthesis of Trigonal Selenium Nanowires. Chem. Mater. 2003, 15, 3852-3858.

(11) Gates, B. B.; Mayers, B.; Cattle, B.; Xia, Y. Synthesis and Characterization of Uniform Nanowires of Trigonal Selenium. Adv. Funct. Mater. 2002, 12, 219-227.

(12) Yan, W.; Qu, Y.; Gupta, T.; Das; Darga, A.; Nguyên, D. T.; Page, A. G.; Rossi, M.; Ceriotti, M.; Sorin, F. Semiconducting
Nanowire-Based Optoelectronic Fibers. Adv. Mater. 2017, 29, 1700681.

(13) Yan, W.; Page, A. G.; Nguyên, D. T.; Qu, Y.; Sordo, F.; Wei, L.; Sorin, F. Advanced Multi-Material Electronic and Optoelectronic Fibers and Textiles. Adv. Mater. 2018.

(14) Bayindir, M.; Abouraddy, A. F.; Sorin, F.; Joannopoulos, J. D.; Fink, Y. Fiber Photodetectors Codrawn From Conducting, Semiconducting and Insulating Materials. Opt. Photonics News 2004, 15, 24.

(15) Sorin, F.; Lestoquoy, G.; Danto, S.; Joannopoulos, J. D.; Fink, Y. Resolving Optical Illumination Distributions along an Axially Symmetric Photodetecting Fiber. Opt. Express 2010, 18, 2426424275.

(16) Jackson, P.; Hariskos, D.; Lotter, E.; Paetel, S.; Wuerz, R.; Menner, R.; Wischmann, W.; Powalla, M. New World Record Efficiency for $\mathrm{Cu}(\mathrm{In}, \mathrm{Ga}) \mathrm{Se}_{2}$ thin-Film solar Cells beyond 20\%. Prog. Photovoltaics 2011, 19, 894-897.

(17) Siebentritt, S.; Schorr, S. Kesterites-a Challenging Material for Solar Cells. Prog. Photovoltaics 2012, 20, 512-519.

(18) Kasap, S.; Frey, J. B.; Belev, G.; Tousignant, O.; Mani, H.; Greenspan, J.; Laperriere, L.; Bubon, O.; Reznik, A.; DeCrescenzo, G.; et al. Amorphous and Polycrystalline Photoconductors for Direct Conversion Flat Panel X-Ray Image Sensors. Sensors 2011, 11, 51125157.

(19) Yan, W.; Nguyen, T. D.; Cayron, C.; Dasgupta, T.; Page, A. G.; $\mathrm{Qu}$, Y.; Sorin, F. Microstructure Tailoring of Selenium-Core Multimaterial Optoelectronic Fibers. Opt. Mater. Express 2017, 7, $1388-1397$.

(20) Ray, C.; Dutta, S.; Sarkar, S.; Sahoo, R.; Roy, A.; Pal, T. A Facile Synthesis of 1D Nano Structured Selenium and Au Decorated Nano Selenium: Catalysts for the Clock Reaction. RSC Adv. 2013, 3, 24313-24320.

(21) Dong, H.; Quintilla, A.; Cemernjak, M.; Popescu, R.; Gerthsen, D.; Ahlswede, E.; Feldmann, C. Colloidally Stable Selenium@copper Selenide Core@shell Nanoparticles as Selenium Source for Manufacturing of Copper-Indium-Selenide Solar Cells. J. Colloid Interface Sci. 2014, 415, 103-110.

(22) Deng, D. S.; Orf, N. D.; Danto, S.; Abouraddy, A. F.; Joannopoulos, J. D.; Fink, Y. Processing and Properties of Centimeter-Long, in-Fiber, Crystalline-Selenium Filaments. Appl. Phys. Lett. 2010, 96, 023102.

(23) Yaman, M.; Khudiyev, T.; Ozgur, E.; Kanik, M.; Aktas, O.; Ozgur, E. O.; Deniz, H.; Korkut, E.; Bayindir, M. Arrays of Indefinitely Long Uniform Nanowires and Nanotubes. Nat. Mater. 2011, 10, 494-501.

(24) Wang, C.; Hu, Q.; Wei, Y.; Fang, D.; Xu, W.; Luo, Z. Facile Fabrication of Selenium (Se) Nanowires for Enhanced Lithium Storage in Li-Se Battery. Ionics 2017, 23, 3571-3579.

(25) Zhang, J.; Xu, Y.; Fan, L.; Zhu, Y.; Liang, J.; Qian, Y. GrapheneEncapsulated Selenium/polyaniline Core-Shell Nanowires with Enhanced Electrochemical Performance for Li-Se Batteries. Nano Energy 2015, 13, 592-600.

(26) Chaudhary, S.; Umar, A.; Mehta, S. K. Selenium Nanomaterials: An Overview of Recent Developments in Synthesis, Properties and Potential Applications. Prog. Mater. Sci. 2016, 83, 270-329.

(27) Cao, X. B.; Xie, Y.; Zhang, S. Y.; Li, F. Q. Ultra-Thin Trigonal Selenium Nanoribbons Developed from Series-Wound Beads. Adv. Mater. 2004, 16, 649-653.

(28) Abdelouas, A.; Gong, W. L.; Lutze, W.; Shelnutt, J. A.; Franco, R.; Moura, I. Using Cytochrome c3 to Make Selenium Nanowires. Chem. Mater. 2000, 12, 1510-1512.

(29) Wang, M. C. P.; Zhang, X.; Majidi, E.; Nedelec, K.; Gates, B. D. Electrokinetic Assembly of Selenium and Fibers. ACS Nano 2010, 4, 2607-2614.

(30) Karpowicz, N.; Dai, J.; Lu, X.; Chen, Y.; Yamaguchi, M.; Zhao, H.; Zhang, X. C.; Zhang, L.; Zhang, C.; Price-Gallagher, M.; et al. Coherent Heterodyne Time-Domain Spectrometry Covering the Entire "Terahertz Gap. Appl. Phys. Lett. 2008, 92, 011131. 
(31) Cherin, P.; Unger, P. The Crystal Structure of Trigonal Selenium. Inorg. Chem. 1967, 6, 1589-1591.

(32) Dantzig, J. A.; Rappaz, M. Solidification; EPFL Press: Lausanne; Taylor and Francis: London, 2009.

(33) Suslick, K. S. Sonochemistry. Science 1990, 247, 1439-1445.

(34) Lingelbach, W.; Weiser, G.; et al. Temperature-Dependent Electroabsorption on the Indirect Edge of Trigonal Selenium. Phys. Rev. B 1972, 5, 243-253.

(35) Matsui, M. Role of Interchain Interaction in Determining the Band Gap of Trigonal Selenium: A Density Functional Theory Study with a Linear Combination of Bloch Orbitals. J. Phys. Chem. C 2014, 118, 19294-19307.

(36) Fountaine, K. T.; Whitney, W. S.; Atwater, H. A. Resonant Absorption in Semiconductor Nanowires and Nanowire Arrays: Relating Leaky Waveguide Modes to Bloch Photonic Crystal Modes. J. Appl. Phys. 2014, 116, 153106.

(37) Rai, R. C. Analysis of the Urbach Tails in Absorption Spectra of Undoped ZnO Thin Films. J. Appl. Phys. 2013, 113, 153508.

(38) Ulbricht, R.; Hendry, E.; Shan, J.; Heinz, T. F.; Bonn, M. Carrier Dynamics in Semiconductors Studied with Time-Resolved Terahertz Spectroscopy. Rev. Mod. Phys. 2011, 83, 543-586.

(39) Joyce, H. J.; Baig, S. A.; Parkinson, P.; et al. A Review of the Electrical Properties of Semiconductor Nanowires: Insights Gained from Terahertz Conductivity Spectroscopy The Influence of Surfaces on the Transient Terahertz Conductivity and Electron Mobility of GaAs Nanowires. Semicond. Sci. Technol. 2016, 31, 103003.

(40) Johnston, M. B.; Herz, L. M. Hybrid Perovskites for Photovoltaics: Charge-Carrier Recombination, Diffusion, and Radiative Efficiencies. Acc. Chem. Res. 2016, 49, 146-154.

(41) Manser, J. S.; Kamat, P. V. Band Filling with Free Charge Carriers in Organometal Halide Perovskites. Nat. Photonics 2014, 8, 737-743.

(42) Knab, J. R.; Lu, X.; Vallejo, F. A.; Kumar, G.; Murphy, T. E.; Hayden, L. M. Ultrafast Carrier Dynamics and Optical Properties of Nanoporous Silicon at Terahertz Frequencies. Opt. Mater. Express 2014, 4, 300-307.

(43) Zheng, K.; Žídek, K.; Abdellah, M.; Chen, J.; Chábera, P.; Zhang, W.; Al-Marri, M. J.; Pullerits, T. High Excitation Intensity Opens a New Trapping Channel in Organic-Inorganic Hybrid Perovskite Nanoparticles. ACS Energy Lett. 2016, 1, 1154-1161.

(44) Bouduban, M. E. F.; Burgos-Caminal, A.; Ossola, R.; Teuscher, J.; Moser, J.-E. Energy and Charge Transfer Cascade in Methylammonium Lead Bromide Perovskite Nanoparticle Aggregates. Chem. Sci. 2017, 8, 4371-4380.

(45) Aleithan, S. H.; Livshits, M. Y.; Khadka, S.; Rack, J. J.; Kordesch, M. E.; Stinaff, E. Broadband Femtosecond Transient Absorption Spectroscopy for a CVD $\mathrm{MoS}_{2}$ Monolayer. Phys. Rev. B: Condens. Matter Mater. Phys. 2016, 94, 035445.

(46) Lou, Y.; Chen, X.; Samia, A. C.; Burda, C. Femtosecond Spectroscopic Investigation of the Carrier Lifetimes in Digenite Quantum Dots and Discrimination of the Electron and Hole Dynamics via Ultrafast Interfacial Electron Transfer. J. Phys. Chem. B 2003, 107, 12431-12437.

(47) Cooper, W. C. The Physics of Selenium and Tellurium, $2^{\text {nd }}$ ed.; Elsevier: New York, 1969.

(48) Shockley, W.; Read, W. T. Statistics of the Recombination of Holes and Electrons. Phys. Rev. 1952, 87, 835-842.

(49) Bhaskar, P.; Achtstein, A. W.; Diedenhofen, S. L.; Siebbeles, L. D. A. Mobility and Decay Dynamics of Charge Carriers in OneDimensional Selenium van Der Waals Solid. J. Phys. Chem. C 2017, 121, 18917-18921.

(50) Uhd Jepsen, P.; Schairer, W.; Libon, I. H.; Lemmer, U.; Hecker, N. E.; Birkholz, M.; Lips, K.; Schall, M. Ultrafast Carrier Trapping in Microcrystalline Silicon Observed in Optical Pump-Terahertz Probe Measurements. Appl. Phys. Lett. 2001, 79, 1291-1293.

(51) Wehrenfennig, C.; Liu, M.; Snaith, H. J.; Johnston, M. B.; Herz, L. M. Charge-Carrier Dynamics in Vapour-Deposited Films of the Organolead Halide Perovskite $\mathrm{CH}_{3} \mathrm{NH}_{3} \mathrm{PbI}_{3-\mathrm{x}} \mathrm{Cl}_{\mathrm{x}}$. Energy Environ. Sci. 2014, 7, 2269-2275.
(52) Liao, Z. M.; Hou, C.; Zhao, Q.; Liu, L. P.; Yu, D. P. Gate Tunable Photoconductivity of P-Channel Se Nanowire Field Effect Transistors. Appl. Phys. Lett. 2009, 95, 1-4.

(53) Mort, J. Transient Photoconductivity in Trigonal Selenium Single Crystals. J. Appl. Phys. 1968, 39, 3543-3549.

(54) Baxter, J. B.; Schmuttenmaer, C. A. Conductivity of $\mathrm{ZnO}$ Nanowires, Nanoparticles, and Thin Films Using Time-Resolved Terahertz Spectroscopy. J. Phys. Chem. B 2006, 110, 25229-25239. 
Supporting Information for

\section{Direct Synthesis of Selenium Nanowire Mesh}

on a Solid Substrate and Insights into Ultrafast Photocarrier Dynamics

Wei Yan, ${ }^{\dagger}, \#$ Andrés Burgos-Caminal, ${ }^{+,}$, , Tapajyoti Das Gupta, ${ }^{\dagger}$ Jacques-E. Moser, ${ }^{+}$

\section{Fabien Sorin t, *}

${ }^{\dagger}$ Laboratory of Photonic Materials and Fibre Devices (FIMAP), Institute of Materials, École polytechnique fédérale de Lausanne, CH-1015 Lausanne, Switzerland

$\$$ Photochemical Dynamics Group, Institute of Chemical Sciences \& Engineering and Lausanne Centre for Ultrafast Science (LACUS), École polytechnique fédérale de Lausanne, CH-1015 Lausanne, Switzerland

The NFP results were fitted using standard exponential equations:

$$
S(t)=A \exp \left(-\frac{t-t_{0}}{\tau}\right)
$$

The equation used to fit the TRTS charge carrier dynamics is the result of the convolution between a Gaussian and an exponential equation (2) due to the short timescale of the decay.

$$
S(t)=A \frac{1}{2} \exp \left(\frac{\sigma^{2}}{2 \tau^{2}}-\frac{t-t_{0}}{\tau}\right)\left(1+\operatorname{erf}\left(\frac{t-t 0}{\sqrt{2} \sigma}-\frac{\sigma}{\sqrt{2} \tau}\right)\right)
$$


Considering an instrument response in the form of the following Gaussian for the convolution:

$$
f(t)=\frac{1}{\sigma \sqrt{2 \pi}} e^{-\frac{(t-t 0)^{2}}{2 \sigma^{2}}}
$$

\begin{tabular}{|c|c|c|c|c|c|c|}
\hline $\begin{array}{c}\text { Fluence } / \mu \mathrm{J} \\
\mathrm{cm}^{-2}\end{array}$ & $\tau_{1} / \mathrm{ps}$ & $\tau_{2} / \mathrm{ps}$ & $\tau_{3} / \mathrm{ps}$ & $\mathrm{A}_{1}$ & $\mathrm{~A}_{2}$ & $\mathrm{~A}_{3}$ \\
\hline 71 & 0.68 & 4.67 & 1172 & $\begin{array}{c}2.00 \cdot 10^{-} \\
2\end{array}$ & $\begin{array}{c}8.10 \cdot 10^{-} \\
3\end{array}$ & $\begin{array}{c}2.40 \cdot 10^{-} \\
3\end{array}$ \\
\hline 142 & 0.7 & 5.18 & 827 & $\begin{array}{c}2.52 \cdot 10^{-} \\
2\end{array}$ & $\begin{array}{c}1.17 \cdot 10^{-} \\
2\end{array}$ & $\begin{array}{c}4.10 \cdot 10^{-} \\
3\end{array}$ \\
\hline 227 & 0.46 & 3.03 & 241 & $\begin{array}{c}5.32 \cdot 10^{-} \\
2\end{array}$ & $\begin{array}{c}2.30 \cdot 10^{-} \\
2\end{array}$ & $\begin{array}{c}4.10 \cdot 10^{-} \\
3\end{array}$ \\
\hline 284 & 0.5 & 3.69 & 450 & $\begin{array}{c}6.94 \cdot 10^{-} \\
2\end{array}$ & $\begin{array}{c}3.03 \cdot 10^{-} \\
2\end{array}$ & $\begin{array}{c}4.00 \cdot 10^{-} \\
3\end{array}$ \\
\hline 426 & 0.44 & 2.68 & 28 & $\begin{array}{c}8.32 \cdot 10^{-} \\
2\end{array}$ & $\begin{array}{c}4.46 \cdot 10^{-} \\
2\end{array}$ & $\begin{array}{c}8.60 \cdot 10^{-} \\
3\end{array}$ \\
\hline 568 & 0.42 & 2.53 & 32 & $\begin{array}{c}9.20 \cdot 10^{-} \\
2\end{array}$ & $\begin{array}{c}4.96 \cdot 10^{-} \\
2\end{array}$ & $8.3 \cdot 10^{-3}$ \\
\hline
\end{tabular}

Table S1 Fluence dependent TRTS measurements of carrier lifetime. The data were fitted using a triexponential version of equation 2 .

\begin{tabular}{|c|c|c|}
\hline $\begin{array}{c}\text { Fluence }(\mathrm{mJ} \\
\left.\mathrm{cm}^{-2}\right)\end{array}$ & $\tau_{1} / \mu \mathrm{s}$ & $\tau_{2} / \mu \mathrm{s}$ \\
\hline 83 & 1.2 & 4.6 \\
\hline 123 & 0.73 & 3.8 \\
\hline 211 & 0.69 & 3.7 \\
\hline
\end{tabular}

Table S2 Fluence dependent NFP measurements of carrier lifetime. The data were fitted using a biexponential version of equation 1 . 